\title{
Bibliotecas digitales y sociedad de la información
}

\author{
María del Carmen Agustín Lacruz \\ Universidad de Zaragoza \\ Ciencias de la Documentación e Historia de la Ciencia
}

\subsection{Resumen}

La nueva institución documental emergente a la que se le asignan denominaciones como biblioteca electrónica, biblioteca virtual, biblioteca digital, biblioteca universal y hasta biblioteca global — que a pesar de no serlo, se utilizan de hecho como sinónimas-se situa dentro del contexto tecnológico, científico, económico y social en el que surge. Se muestra como la biblioteca digital es generada y se incardina dentro de la urdimbre que le procura el nuevo marco de referencia que convenimos en denominar sociedad de la información. Se identifican y analizan desde una perspectiva procesual los mecanismos de transformación que se producen dentro de la biblioteca pública. (Autor)

Palabras clave: Sociedad de la información. Biblioteca Pública. Biblioteca electrónica. Biblioteca virtual. Biblioteca digital.

\subsection{Abstract}

The new documentation institution to which names as electronic library, virtual library, digital library, universal library and even global library have been given - which, not being synonyms, are used as if they were - is situated in the technological, scientific, economic and social context where it emerges: the information society. This is the context that explains the transformations that the public library is suffering. Such processes of change are identified and analysed.

Keywords: Information society. Public libraries. Electronic libraries. Virtual libraries. Digital libraries.

\section{La biblioteca pública: una institucion que interactua con el entorno}

La biblioteca es una institución documental que en la actualidad sufre una importante crisis de identidad que amenaza incluso, según las voces más agore- 
ras, con su desaparición. Su existencia reposada se ha visto sacudida en los últimos decenios por importantes cambios cuantitativos y cualitativos. Estos procesos de transformación son la respuesta que la biblioteca, una institución tradicionalmente dialogante con el medio en el que se desarrolla y con las necesidades de información planteadas por los usuarios de sus servicios, intenta ofrecer en el nuevo contexto tecnológico, científico, económico y social con el que encaramos el tercer milenio.

El objetivo de este trabajo es situar la nueva institución documental emergente dentro del marco de referencia que le proporciona la sociedad de la información. Aclararemos términos como biblioteca electrónica, biblioteca virtual, biblioteca digital, biblioteca universal y biblioteca global, utilizados con profusión en la bibliografía actual de forma sinónima o cuasi sinónima, cuando en realidad no lo son, e intentaremos identificar los procesos de transformación que se dan en el seno de la institución bibliotecaria, señalando las funciones que permanecen y las nuevas estructuras y servicios que las tecnologías de la información posibilitan.

La biblioteca digital, desde esta perspectiva, es nada más y nada menos que la respuesta a la interacción que con su entorno y con las necesidades de sus usuarios establece la institución documental que conocemos como biblioteca pública, en el contexto de la sociedad de la información. Ambos conceptos están ligados: la biblioteca digital surge dentro de la sociedad de la información, y ésta para desarrollarse de forma democrática y sostenible, necesita una biblioteca pública vigorosa, actual, operativa y al alcance de todos.

\section{La sociedad de la información. Un nuevo paradigma}

La Sociedad de la Información constituye el contexto genérico en el que surgen y cobran sentido las nuevas formas que adopta la biblioteca, por eso comenzaremos definiéndola.

Todas las sociedades actuales, con independencia de su grado de desarrollo, o de su orientación política, avanzadas o en vías de desarrollo, otorgan un gran valor al fenómeno informativo. No en balde, la información, sea cual sea el ámbito temático del que se ocupe, es punto de partida imprescindible para llevar a cabo con eficacia y prontitud cualquier actividad humana.

Como consecuencia de los avances tecnológicos de las últimas décadas nos hemos adentrado en un nuevo paradigma tecnológico, económico y social que diferentes especialistas coinciden en denominar informacional o sociedad de la información, y que representa una divisoria histórica similar a la que constituyó en su momento la Revolución Industrial. 
Los orígenes de la sociedad de la información descansan sobre dos tipos de fenómenos interdependientes: el desarrollo económico a largo plazo y la evolución tecnológica.

Por lo que respecta al primero de los dos factores, hay que señalar que la estructura y evolución económicas se caracterizan porque, en un principio, toda la actividad reposa sobre el sector primario (agricultura, ganadería, minería, silvicultura), progresivamente, la fuerza productiva se desplaza hacia el sector secundario (industria y manufacturas) y finalmente, el crecimiento del sector secundario es seguido por la expansión del sector terciario (comercio y servicios). Todos los países occidentales han ido pasando por los diferentes estadios de este proceso a lo largo del último milenio, y en los últimos cincuenta años, el sector servicios se ha orientado cada vez más hacia el tratamiento de la información, bajo sus diferentes formas.

La evolución tecnológica, esto es, el segundo de los factores en liza, ha contribuido ampliamente al desarrollo de este proceso económico, pues resulta indudable que el rápido desarrollo que han experimentado las tecnologías de la información y de las telecomunicaciones ha incrementado notablemente nuestra capacidad de tratamiento de la información, acelerando el crecimiento del sector terciario, consumidor indudable de información (Moore, 1997).

La actual transformación tecnológica está basada sobre las tecnologías de la información: microelectrónica, informática, telecomunicaciones, inteligencia artificial e ingeniería genética (Castells, 1995), y su impacto social se deriva de tres de sus características (Moore, 1997):

- Es una tecnología que proporciona medios, es aplicable a toda clase de situaciones, y ella misma contribuye a la evolución tecnológica.

- Sus capacidades aumentan, desde hace una veintena de años, según un ritmo de crecimiento exponencial, y ningún indicador marca que este ritmo vaya a detenerse.

- Sus costes disminuyen rápidamente, y este fenómeno, extraño en un principio, parece que esta llamado a durar.

Este conjunto de hechos motiva que algunos economistas y analistas sociales señalen que las tecnologías de la información y las comunicaciones están desencadenando una nueva ola de crecimiento económico, que lleva en sí un nuevo estímulo para el desarrollo de la sociedad de la información.

Indudablemente estos cambios transforman sustancialmente los esquemas tradicionales de empleo, de ocupación del ocio y del tiempo libre, de los hábitos de consumo, y de formación, dando lugar tanto a nuevas formas de desarrollarlos, y a desplazamientos geográficos y sectoriales. 
Pero, ¿qué es la sociedad de la información? En principio, la propia intangibilidad de la información hacen difícil definir y describir esta sociedad emergente en términos cuantitativos. Pecando de un exceso de generalismo, podemos señalar que "sociedad de la información es aquella en la que la información (la capacidad del ser humano para manipular, procesar y recuperar datos, informaciones, mensajes, conocimiento, en suma) es utilizada intensivamente en tanto que elemento determinante en la vida económica, social, cultural y política" (Moore, 1997). Según este mismo autor, tres características principales permiten definirla:

- Utiliza la información como un recurso económico. Las empresas utilizan información para incrementar su eficacia, su competitividad, para estimular la innovación y obtener mejores resultados, mejorando así la calidad de los bienes y servicios que producen.

- La información es también un recurso utilizado en gran medida por el gran publico, en tanto que ciudadanos, y en tanto que consumidores, pues el acceso y el uso de la información les permite ejercer sus derechos y responsabilidades cívicas.

- Estas sociedades desarrollan un sector de la información que tiene por función dar respuesta a la demanda general de medios y servicios de información, una parte del cual se relaciona con las infraestructuras tecnológicas (informática y telecomunicaciones) y otra con los creadores de la información - o contenido informativo- que circula por esos recursos.

Este conjunto de características hace que la denominada sociedad de la información se convierta en un fenómeno multidimensional y pluriforme (Canals, 1997) cuyas implicaciones son evidentes en los diferentes órdenes de nuestra vida cotidiana, y cuyos límites resultan difíciles de definir.

La sociedad de la información se articula, o debería articularse, en torno al conocimiento, que deviene en el factor competitivo fundamental. No obstante se constata que aunque uno de los principales efectos de las nuevas tecnologías de la información ha sido acelerar y reducir millones de veces el coste del almacenamiento y la transmisión de la información, no ha ocurrido lo mismo respecto a la generación de conocimientos, y aun menos de saber. La sociedad de la información tiene que tender hacia una sociedad del conocimiento, hacia una sociedad sabia, que permita configurar una sociedad sostenible, que contemple el bienestar de todos sus miembros.

Por eso es importante percibir la sociedad de la información como una sociedad de aprendizaje continuo, de formación permanente en nuevas habilidades.

Las instituciones documentales han sido a lo largo de su historia organizadoras y transmisoras eficientes de este conocimiento. Hoy la revolución digital hace 
que el modo en que tratamos la información portada documentalmente adquiera una posición nuclear, pues determina, no solo un nuevo modo de transmisión de la información, sino que lleva consigo una propuesta de nuevos objetivos, nuevas funciones y nuevos modelos organizativos.

\section{De la mediateca a la biblioteca digital}

Estos hechos inciden de forma determinante sobre las diferentes instituciones que tradicionalmente han llevado a cabo las tareas de colecta, conservación y difusión del conocimiento portado y transmitido documentalmente. De forma especial comportan notables modificaciones en el concepto, organización y en las funciones y los servicios que las bibliotecas han ofrecido tradicionalmente.

A lo largo del tiempo, las bibliotecas públicas han sido las instituciones encargadas de proporcionar acceso, con espíritu democrático, a toda la información generada, garantizando el libre acceso a todos los ciudadanos, ofreciendo oportunidades de educación permanente y salvaguardando la identidad cultural de los pueblos; pero ante los retos tecnológicos que se plantean en la actualidad la mayor parte de las bibliotecas públicas andan aún rezagadas. Para hacer frente al desafío, están en un proceso incipiente de redefinición de su papel y de establecimiento de estrategias de adecuación.

En el contexto de la sociedad de la información el objetivo último de las instituciones bibliotecarias sigue inalterado e inalterable a lo largo del tiempo: ofrecer acceso a cualquier tipo de información, a cualquier usuario que lo precise, en cualquier momento, en cualquier lugar, proporcionándole conjuntos documentales organizados —en cualquier soporte, en átomos o en bits-, que permitan satisfacer sus necesidades informativas. La tecnología actual puede hacer de este propósito una realidad alcanzable, una vez superadas las dependencias y servidumbres impuestas por algunos de los medios y soportes tradicionales.

Para que podamos valorar adecuadamente el estadio evolutivo en el que las bibliotecas se encuentran en la actualidad y vislumbrar así el horizonte más próximo, puede resultar interesante analizar la evolución que la biblioteca ha experimentado en las dos últimas décadas.

\subsection{Concepto y definicion de mediateca}

Aunque en Estados Unidos el fenómeno se produjo diez años antes, en Europa la década de los ochenta se caracterizó por un formidable dinamismo de fuerzas informacionales que fecundaron la biblioteca y amenazaron con hacerla estallar, situación que se refleja en una significativa expresión francesa surgida en este periodo - bibliothèque éclatée - portadora de una carga semántica alusiva a 'estallido con estrépito', es decir ruido, sonido, que se produce 'acompañado 
de esplendor', lo que implica luz, imagen. El impacto se caracterizó por tres manifestaciones principales:

a) La descentralización de la biblioteca, fruto de la generalización de redes y sistemas — fundamentalmente de carácter orgánico y administrativoque coordinaban tareas y compartían recursos.

b) El declive del libro y del papel como vehículos exclusivos de la información "culta".

c) El surgimiento de nuevos medios de transmisión de informaciones y la consiguiente ruptura del monopolio libresco.

En este sentido, la de los ochenta fue la década de la irrupción masiva de diferentes soportes, distintos al papel y al formato códice, creándose una dinámica muy interesante de "reunión/dispersión" de medios (Varet, 1995).

Se generalizó así, por un lado el concepto de biblioteca de medios, mediateca, multiteca, media library o multimedia library, y por otro el de todas y cada una de las tecas especializadas (fototeca, filmoteca, videoteca, diapoteca, iconoteca, fonoteca, etc.).

En este periodo, la multiplicación de las técnicas de comunicación hizo que los otros medios no se viesen unicamente como auxiliares o prolongaciones del libro, sino como auténticos competidores en igualdad de condiciones. Grandes cantidades de información, antes abocadas a la difusión impresa, encuentran otras vías comunicación. La biblioteca asume dentro de sí modos de difusión no librescos y estructuras documentales nuevas. Surge una biblioteca polivalente y múltiple en cuanto a sus soportes - multiteca - que enlaza, a pesar de su aparente novedad, con una tradición histórica muy antigua (1). La heterogeneidad/diversidad se da en los soportes (realidad material), y también en el tipo de información portada (registros sonoros y libros, basados en la oralidad y en la eficacia de lo escrito, imágenes fijas y en movimiento).

De forma paralela nacen las diferentes tecas especializadas en un único medio, de modo que, en lugar de integrarse, se dispersan unos medios de otros en función de las características compartidas. Surgen las artotecas o iconotecas, las cartotecas o mapotecas, las fototecas, las filmotecas, las videotecas, las fonotecas, etc. Cada medio genera su teca específica, pues precisa unas condiciones propias de conservación, unos equipos de lectura, unas técnicas y herramientas de clasificación e indización, y hasta una comunidad de usuarios específica.

En la década de los noventa asistimos a las transformaciones provocadas por el avance imparable de las tecnologías informáticas y las telecomunicaciones. Se produce una eclosión de redes y conectividades, que genera en el ámbito de los servicios de información una ampliación impensable de la capacidad de difusión 


\section{DÉCADA DE LOS 80}

- Ruptura de la primacía del almacenamiento y difusión basada en la eficacia de lo escrito, y lo textual.

- Eclosión de soportes y formatos.

- Institución documental: Mediateca.

- Oposiciones expresivas:

- Escrito/Audiovisual

- Palabra/Imagen

- Papel/Soportes magnéticos

- Formato códice/Otros formatos

\section{DÉCADA DE LOS 90}

- Ruptura de la primacía de la difusión basada en el desplazamiento de átomos.

- Eclosión de redes y conectividades.

- Institución documental: Biblioteca Digital.

- Oposiciones expresivas:

- Real/Virtual.

- Local/Global

- Analógico/Digital

- Texto/Hipertexto

Fig. 1. Procesos experimentados en las bibliotecas públicas. 
de los recursos propios. Emergen nuevas realidades informativas, y surgen nuevos términos para denominarlas (Figura 1).

De forma paralela, el desarrollo de las bibliotecas públicas europeas no es uniforme, se lleva a cabo a diferentes velocidades, y puede dividirse en tres etapas, caracterizadas por el desarrollo de diferentes procesos:

- Automatización de rutinas bibliotecarias básicas (adquisición, registro, catalogación, gestión del préstamo, etc.), es decir, informatización de la gestión mediante equipos y aplicaciones específicas —notablemente abaratados y con unas prestaciones progresivamente mayores. El servicio estrella de esta época fueron los OPACs (2) de primer y segunda generación que proporcionaban acceso público en línea al catálogo.

- Acceso a bases de datos en línea, para el personal del centro y los usuarios. La era de la teledocumentación y las conexiones vía telnet a los OPACs.

- La era Internet. La posibilidad de crear e incorporar servicios basados en servidor de Internet en la página web de la biblioteca, a la que se puede tener acceso remoto.

Cada una de estas etapas corresponde a un estadio de tecnológico distinto. Por tanto, hace referencia a prestaciones, servicios y funciones diversas. A su vez, cada etapa generó su propia denominación diferenciadora.

\section{Precisiones terminológico-conceptuales}

En el momento actual, un importante problema consiste en que la aceleración vertiginosa de los procesos ha hecho que unas denominaciones se superpongan a otras, utilizándose todas como expresiones sinónimas o cuasi-sinónimas, cuando en realidad no lo son. Por eso, es necesario llevar a cabo algunas precisiones de naturaleza terminológica y conceptual.

\subsection{Biblioteca electrónica}

Las bibliotecas que corresponden a la primera fase y por tanto las que reciben una denominación más antigua son las que conocemos como bibliotecas electrónicas (3).

Una biblioteca electrónica es una institución documental que funciona y se organiza como una entidad informativa individual. Proporciona acceso a colecciones controladas de objetos físicos de información, y ha mecanizado sus tareas de gestión y tratamiento documental a través de un programa de automatización (ABSYS, DOBIS/LIBIS, GEAC, LIBER, LOGABIB, OPSYS; ORPHEE, SIBIL, LIBERTAS, SABINI, VTLS, BIBLIO 3000, MDUP, ALEPH, etc). Como cauce de difusión de su fondo documental ha generado catálogos en línea de acceso público, que se constituyen en los servicios emblemáticos del centro. 


\subsection{Biblioteca virtual}

El término virtual ha sido adoptado en los ambientes bibliotecarios procedente de la industria de las computadoras, para significar entornos u objetos informáticos que simulan experiencias como si fuesen reales.

La denominación de biblioteca virtual hace referencia a una colección de documentos electrónicos ( generalmente sedes web) organizados en línea y puestos a disposición de unos usuarios que van a acceder a ellos a través de redes telemáticas, para satisfacer, de este modo, sus necesidades de información a través del ciberespacio (4). Las bibliotecas virtuales carecen de emplazamiento físico real, distinto al de la sede del servidor en que se aloja, por ello su ubicación se describe a través de una URL.

\subsection{Biblioteca digital}

Ligado a los dos términos anteriores y situado en un estadio tecnológico más avanzado, aparece el concepto de biblioteca digital. El adjetivo digital se generaliza en Estados Unidos entre 1993 y 1995 (como se refleja en la base de datos bibliográfica LISA), y se introduce en España de la mano del tecnólogo N. Negroponte en su obra La sociedad digital (1996), donde se señala la oposición exisstente entre los flujos de átomos y los flujos de bits.

Una biblioteca digital, según la definición ofrecida por la Association of Research Libraries, recogida en la Berckeley Digital LibrarySunSITE se define por las siguientes características:

- No es una entidad individual.

- Requiere tecnología para enlazarse a otras.

- Los vínculos entre diferentes bibliotecas digitales y servicios de información son claros para los usuarios y satisfacen sus fines.

- El acceso universal a las bibliotecas digitales y servicios de información es la meta perseguida.

- La colección de la biblioteca digital no se limita a documentos suplentes sino que se extiende a todos los objetos digitales que no pueden ser representados o distribuidos en formato impreso.

Según esta misma Asociación, los propósitos de un sistema de bibliotecas digitales son:

- Facilitar el desarrollo sistemático de los medios para reunir, almacenar y organizar la información y el conocimiento en forma digital.

- Promover la distribución de la información de forma eficiente y económica entre todos los sectores de la sociedad. 


\section{BIBLIOTECA ELECTRÓNICA}

Institución documental que funciona y se organiza como entidad informativa individual

Su objetivo es proporcionar acceso a colecciones controladas de objetos físicos de información

Mecaniza sus tareas de gestión y tratamiento documental a través de programas de automatización

Como cauce de difusión de su fondo documental ha generado catálogos en línea de acceso público, que se constituyen en los servicios emblemáticos del centro.

\section{BIBLIOTECA VIRTUAL}

Colección de documentos digitales (generalmente sedes web)

Su objetivo es satisfacer necesidades de información a través del ciberespacio

Organizados en línea

Puestos a disposición de unos usuarios que acceden a ellos a través de redes telemáticas

Fig. 2. Diferencias entre la biblioteca electrónica y la biblioteca digital

- Alentar esfuerzos cooperativos y promover inversiones dedicadas a recursos de investigación y redes de telecomunicaciones.

- Fortalecer la comunicación y la colaboración entre la comunidad educativa, investigadora, gubernamental y económica.

- Ejercer el liderazgo internacional en la producción y diseminación de conocimiento en áreas de importancia estratégica para América del Norte.

- Contribuir a la creación de oportunidades de aprendizaje para todos los norteamericanos (sic).

Las bibliotecas digitales son bibliotecas en las que las colecciones controladas de información están en forma de objetos digitales, y el acceso a la información se basa en tecnología digital. Respecto de la biblioteca tradicional se han producido varios cambios:

- De la organización de la información basada en estructuras físicas a una organización más flexible basada en estructuras lógicas. 
- De la organización simple y física de la colección hacia una organización múltiple y lógica de la colección.

- Además, los objetos de información en forma digital permiten utilizar tecnología digital para extraer información de ellos. (Smith, 1996).

\subsection{Biblioteca global/biblioteca universal}

Es la biblioteca de la aldea global a la que hacía referencia M. McLuhan, cuyo ámbito físico es el ciberespacio, y cuyo contexto se lo proporciona la cibersociedad. En este sentido, el objetivo de crear una gran y única biblioteca global se vincula con el viejo propósito enciclopédico de recopilar la totalidad del saber, sólo que a través de una organización reticular y compleja.

Un prototipo de desarrollo de este proyecto se localiza en la Carnegie Mellon University de Pittsburgh, Pennsylvania. Esta iniciativa pretende crear una infraestructura técnica y económica que permita ofrecer en Internet acceso a todas las obras de la humanidad, producidas en cualquier lugar y en cualquier tiempo.
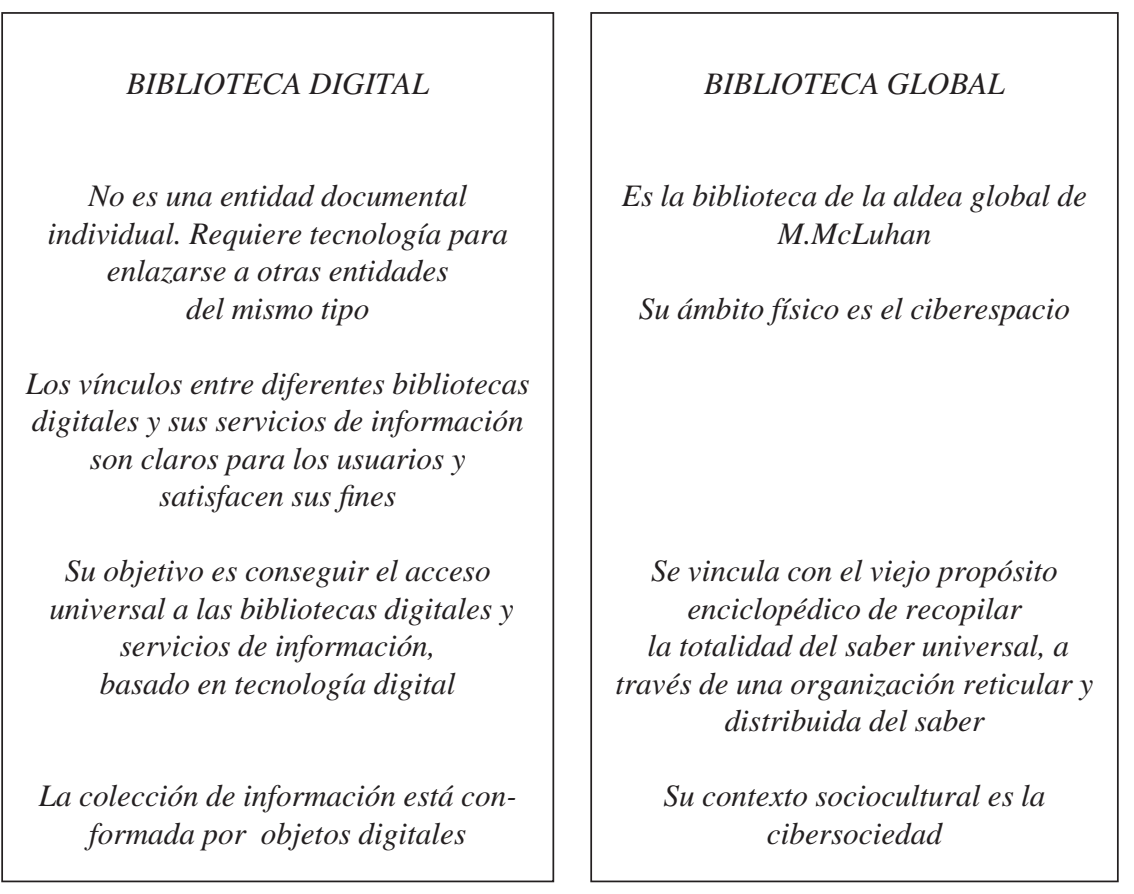

Fig. 3. Diferencias entre la biblioteca digital y la biblioteca global

Scire. $4: 2$ (jul.-dic. 1998) 47-62. 


\section{Conclusiones}

La biblioteca virtual o biblioteca digital implica un recrecimiento, un ensanchamiento notable sobre los servicios y posibilidades de difusión del conocimiento ofrecidos por la biblioteca tradicional. No obstante, en tanto que pervive en ella el espíritu de servicio público, desempeña diferentes funciones nucleares en el proceso de implantación de la sociedad global de la información

- Lleva a cabo una colaboración activa en el mantenimiento de la democracia, pues proporciona un acceso igualitario y sin cortapisas a las fuentes de información generadas.

- Apoya la educación y el aprendizaje en diferentes niveles y estadios educativos, al proporcionar la materia prima del conocimiento: la información.

- Ofrece a los ciudadanos la posibilidad de utilizar nuevas tecnologías de la información, proporcionando el acceso al hardware, al software y a las redes telemáticas, democratizando el acceso telemático al saber.

- Sigue siendo una importante institución cultural vertebradora de la comunidad dentro de la que se inserta y a la que sirve.

No obstante, y a pesar de que nuestra emergente sociedad de la información precisa bibliotecas con las características y funcionalidades que hemos venido señalando, algunos importantes obstáculos dificultan su desarrollo. Estos pueden agruparse en dos categorías:

1. Obstáculos extrabibliotecario/externos:

- Falta de consciencia y/o de confianza política en el potencial de la biblioteca como factor activo dentro de la sociedad de la información

- Falta de planificación administrativa que permita el establecimiento de redes.

- Así mismo, se adolece de una carencia de canales de financiación suficientes así como del necesario apoyo técnico que permita implantar estas nuevas tecnologías.

2. Obstáculos internos:

- Estos nuevos modelos de biblioteca exigen del personal encargado de trabajar en ellas, una actualización profesional permanente, la falta de esta se convierte en una barrera difícil de franquear.

- La falta de visión de futuro y la cortedad de miras de muchos gestores culturales

- La escasa disposición para familiarizarse con nuevas tecnologías.

- Los miedos y reticencias ante los cambios de concepción de la función y los servicios que el nuevo modelo de biblioteca debe ofrecer. 
- Escasez de profesionales competentes en tecnologías de la información.

Estos últimos factores merecen un comentario adicional. La evolución tecnológica y el desarrollo de las tecnologías de la información ha provocado un fuerte impacto sobre los profesionales de la información que desempeñan sus tareas en centros de documentación, bibliotecas, archivos, servicios y centros de información, etc. Estos profesionales sufren dos presiones complementarias: por una parte, las tecnologías han extendido notablemente su campo de actividad; y, por otro lado, las expectativas crecientes de los usuarios generan una demanda de servicios de información cada vez más sofisticada y de gran calidad.

Estas presiones exigen que los profesionales estén cada vez más cualificados y que posean las competencias necesarias para explotar plenamente el potencial tecnológico. Además, su curriculum profesional, a diferencia de lo que acontecía hace unas decenas de años, ha de ser completado con una formación continuada que desarrolle, actualice y perfeccione sus competencias profesionales. En los últimos años ha surgido un nuevo tipo de profesional provisto de nuevas cualificaciones: el webmaster, responsable de la creación, mantenimiento y actualización de los sitios web. Es, en parte, profesional de la información; y, en parte, especialista en edición electrónica, diseñador y experto en informática.

¿Es acaso el webmaster el nuevo modelo de profesional de la información? Tenga esta pregunta una respuesta afirmativa o negativa, es indudable que las bibliotecas digitales requieren bibliotecarios digitales, que seleccionen, adquieran, organicen, hagan accesibles y mantengan las colecciones. Esta realidad constituye un importante reto para los propios profesionales en ejercicio y también para las instituciones, académicas y no académicas, que se encargan de formarlos.

\section{Notas}

(1) Desde el Renacimiento y durante los siglos XVI y XVII las bibliotecas estaban asociadas a colecciones pictóricas y museográficas. En ellas era posible encontrar vasos griegos, medallones, globos terrestres y celestes, retratos y bustos (Jolly, 1998; Escolar, 1987).

(2) Veáse al respecto el interesante y clarificador capítulo de F. J. García Marco (1996).

(3) Veáse a este respecto Carrión (1998) y Amat (1990)

(4) El término ciberespacio fue acuñado en 1984 por William Gibson en su novela Neouromancer (1997) haciendo referencia al nacimiento de la cultura de la realidad virtual en la que aparecen grandes redes de ordenadores que recrean un mundo futuro. A partir del término cibernética, empleado con el significado de "todos los aspectos relativos a los mecanismos de cálculo y control", el prefijo cyber pierde su significado original de piloto o timonel, para utilizarse en todas las actividades en las que

Scire. $4: 2$ (jul.-dic. 1998) 47-62. 
intervienen las nuevas tecnologías de la información: ciberespacio, cibersociedad, cibercultura, etc. De esta forma, el ciberespacio se define como el espacio que se crea cuando se establece una conexión telemática entre dos o más máquinas (ordenadores, teléfonos, faxes, tarjetas de crédito electrónicas, etc.). El ciberespacio es también, retomando la metáfora de McLuhan, el espacio universal de la aldea global (Rodriguez Baena, 1997, 35-36). Para la autora es revelador el hecho de que, en los albores del tercer milenio, cuando las redes telemáticas están modificando la percepción que tenemos del espacio exterior, acudamos a un genero de ficción para tomar prestado un nombre con el que delimitar y asimilar las novedades sorprendentes, de la misma manera que en la época del descubrimiento de América, cuando el horizonte del mundo conocido se amplió sustancialmente, lo imaginario de raíz literaria guiaba al conquistador y le permitía explicarse y denominar lo desconocido, así la denominación del río Amazonas, el topónimo de California procedente del libro de caballerías Las sergas de Esplandián de Rodríguez de Montalvo, 1510, y el de Patagonia, o tierra habitada por los patagones, a imitación del gigante denominado Gran patagón que aparece en el Primaleón. Véase la interesantísima y deliciosa obra de Lacarra y Cacho Blecua (1990).

\section{Bibliografía}

An architecture for Information in Digital Libraries. // D-Lib Magazine. (February, 1997). URL=<http://www.dlib.org/dlib/february/cnri/02arms1.html >.

Arms, C. R. (1996). Historical Collections for the National Digital Library. // D-Lib Megazine. April, 1996. URL=<http://www.dlib.org./dlib/april96/loc/04carms.html>.

Arms, W. Y. (1995). Key Concepts in the Architecture of the Digital Library. //D-Lib Magazine. (July, 1995). URL=<http://www.cnri.reston.va.us/home/dlib/ July95/07arms.thml>.

Arms, W. Y. (1997). Relaxing Assumptions about the Future of Digital Libraries. // D-Lib Magazine. (April 1997). URL=<http://www.dlib.org/dlib/apri197/04arms.html>.

Bibliotecas públicas y la sociedad de la información : un estudio. $\mathrm{URL}=<$ http://www2.echo.lu/libraries/es/plis/study.html $>$.

Birmingham, W. P. (1995). An Agent-Based Architecture for Digital Libraries.//D-Lib Magazine. (July, 1995). URL=<http://www.dlib.org/dlib/July95/ 07birmingham.html>.

Borgman, Ch. L. (1997). Multi-Media, Multi-Cultural, and Multi-Lingual Digital Libraries : Or How Do We Exchange Data In 400 Languages?. // D-Lib Magazine. June, 1997. URL=<http://www.dlib.org/dlib/june97/06borgman.html>.

Bustamante Donas, J. (1997). La revolución del conocimiento y la nueva cultura digital. // Documentación social : Revista de estudios sociales y de sociología aplicada. 108 (Julio-Septiembre 1997) 179-202.

Canals Cabiró, I. (1997). La societat de la informació en 34 punts i una esperança. // Cid Leal, P. ; Baró i Queeralt, J. (Eds.). Anuari SOCADI de Documentació i Informació. Barcelona : Societat Catalana de Documentació i Informació, 1997. P.153-160. 
Cardona de Gil, B. N. (1996). Nuevos paradigmas para el acceso y uso de la información: la biblioteca virtual. // Revista Interamericana de Bibliotecología. 19 : 1 ( EneroJunio 1996) 31-52.

Carrión Gutiez, M. (1990). Manual de bibliotecas . Madrid ; Salamanca: Pirámide ; F.G.S.R., 1988, pag. 685-695, también Amat i Nogera, N. La biblioteca electrónica. . Madrid; Salamanca: Pirámide; F.G.S.R., 1990.

Castells, M. (1997). La era de la información: Economía, sociedad y cultura : Vol. 1 : La sociedad red. Madrid : Alianza Editorial, 1997.

Castells, M. (1998). La era de la información : Economía, sociedad y cultura : Vol. 2 : El poder de la identidad. Madrid : Alianza Editorial, 1998.

Digital Libraries. URL=<http://info.lib.uh.edu/sepb/Ibdiglib.html >.

Dykstra, M. (1997). Les autoroutes de l'information. // Courier, Y. (Dir.). Rapport mondial sur l'information 1997/1998. París : UNESCO, 1997. P.302-323.

ECSC-EC-EAEC. Las bibliotecas públicas y la sociedad de la información: un estudio. Bruselas ; Luxemburgo, 5 de mayo 1997. URL=<http://www2.echo.lu/libraries/es/ plis/study.html>

Escolar, H. (1987). Historia de las bibliotecas. Madrid ; Salamanca: Pirámide ; F.G.S.R., 1987.

From the editor. // D-Lib Magazine. (October, 1997). URL=<http://www.dlib.org/ dlib/october97/10editorial.html>.

García Marco, F. J (1996). Los catálogos automatizados y su consulta pública. // Orera Orera, L.(ed.) Manual de Biblioteconomía. Madrid : Síntesis, 1996. P. 153-179.

Gibson, William (1997). Neouromante. Madrid : Minotauro, 1997.

Hastings, K.; Tennant, R.(1996). How to Build a Digital Librarian. // D-Lib Magazine. (November, 1996). URL=<http://www.dlib.org/dlib/november96/ucb/ 11hastings.html>.

Hearst, M. A. (1996). Research in Support of Digital Libraries at Xerox PARC. // D-Lib Magazine. (May, 1996). URL=<http://www.dlib.org/dlib/may96/05hearts.html>.

Jolly, C. (ed.) (1998). Histoire des bibliothèques françaises : II: Les bibliothèques sur l’Ancient Régime. Paris : Promodis, 1988.

Joyanes Aguilar, L. (1997). La galaxia Internet : La última utopía : Condionantes y apuestas. // Documentación social : Revista de estudios sociales y de sociología aplicada. 108 : (Julio-Septiembre 1997) 71-102.

Lacarra, M .J. ; Cacho Blecua, J. M. (1997). Lo imaginario en la conquista de América. Zaragoza: Comisión Aragonesa del Quinto Centenario ; D.G.A., 1990.

Lorente, S. (1997). La Sociedad de la Información : su imagen, su proceso, sus logros y sus inconvenientes. // Documentación social : Revista de estudios sociales y de sociología aplicada. 108 (Julio-Septiembre 1997) 155-178.

Martín García, V. (1997). La Edad de la Informática : La cibersociedad.// Documentación social: Revista de estudios sociales y de sociología aplicada. 108 (Julio-Septiembre 1997) 11-32. 
Martínez Comeche, J.A. (1995). Teoría de la información documental y de las instituciones documentales. Madrid: Síntesis, 1995.

Masullo, M. ; Mack, R. (1996). Roles for digital Libraries in K-12 Education. // D-Lib Megazine. (September, 1996). URL=<http://www.dlib.org/dlib/september96/eduport/09masullo.html>.

Moore, N. (1997). La société de l'information. // Courier, Y. (Dir.). Rapport mondial sur l'information 1997/1998. París : UNESCO, 1997. P. 289-302.

Rodríguez Baena, L. (1997). Ciberespacio, cibercultura y realidad virtual. // Documentación social : Revista de estudios sociales y de sociología aplicada. 108 (Julio-Septiembre 1997) 33-50.

Roiz, M. (1997). Algunas consecuencias sociales de la implantación de las nuevas tecnologías de la información y comunicación a finales del siglo XX. // Documentación social : Revista de estudios sociales y de sociología aplicada. 108 (Julio-Septiembre 1997) 257-270.

Smith, T.R.(1996). The Meta-Information Environment of Digital Libraries. // D-Lib Megazine. (July-August, 1996).

Towards the Millennium. // Digital Libraries'98 : The Third ACM Conference on Digital Libraries. Pittsburg, PA, USA, June 23-26, 1998. URL=<http://www.ks.com/d198cfp-long-version.html>.

Trejo Delarbre, R. (1996). La nueva alfombra mágica. Madrid: FUNDESCO, 1996.

The Universal Library. URL=<http://www.ul.cs.cmu.edu/first.html〉.

Varet, G. ; Varet, M. M. (1995). Maitriser l'information a travers sa terminologie. Paris: Universitè de Franche-Comtè, 1995. (Annales Littéraires de l'Université de Besançon ; 559). 